\title{
Does the Tendency to Thrombosis Increase in Autoimmune Thyroid Dysfunction? Rotational Thromboelastometric Analysis
}

Güven Barış Cansu ( $\square$ bcansu74@hotmail.com )

Kütahya Health Sciences University https://orcid.org/0000-0003-2606-1103

Döndü Üsküdar Cansu

Eskisehir Osmangazi University

\section{Bengür Taşkıran}

Kütahya Health Sciences University

Hava Üsküdar Teke

Eskişehir Osmangazi University

\section{Research Article}

Keywords: autoimmune thyroid disease, hyperthyroidism, hypothyroidism, thrombosis,

thromboelastography

Posted Date: February 1st, 2022

DOI: https://doi.org/10.21203/rs.3.rs-1286551/v1

License: (1) (1) This work is licensed under a Creative Commons Attribution 4.0 International License. Read Full License 


\section{Abstract}

The tendency for thrombosis is a well-known issue in hyperthyroidism, and the tendency for both bleeding and thrombosis has been shown in hypothyroidism. In the study, we aimed to establish the tendency to thrombosis by evaluating the coagulation system in thyroid diseases using thromboelastography (TEG).

Thirty patients with new-onset autoimmune-based hypothyroidism, 30 with hyperthyroidism and 30 healthy volunteer subjects were enrolled in this study. Complete blood count, prothrombin time, activated partial thromboplastin time, fibrinogen levels and rotational thromboelastography (ROTEM) were studied. In the ROTEM/TEG analysis, coagulation time (CT), coagulation formation time (CFT) and maximum clot firmness (MCF) were measured. Short CT and/or CFT and/or long MCF were presumed as the tendency to hypercoagulability.

The mean age of subjects with hyperthyroidism and hypothyroidism was $46 \pm 15.9$ years and $44.8 \pm 13$ years, respectively. The thrombocyte count in the hypothyroid group and both thrombocyte counts and fibrinogen levels in the hyperthyroid group was significantly higher than in the healthy group $(p=0.004, p=0.001$, $p<0.001$, respectively). According to ROTEM/TEG results, higher mean MCF figures were noted in both the hypo- and hyperthyroid groups compared with the healthy group ( $p=0.014$ (hypothyroid vs. healthy groups), $p=0.020$ (hyperthyroid vs. healthy groups)).

We showed a tendency for thrombosis using ROTEM in patients with both hyper- and hypothyroidism without thrombosis. The correlation between ROTEM/TEG parameters and the main components of thrombosis formation (thrombocyte count and fibrinogen level) indicate that the main factor might be inflammation (autoimmunity), apart from thyroid hormone levels.

\section{Introduction}

Thyroid dysfunction, either as hypothyroidism or hyperthyroidism, may lead to hemostatic abnormalities varying from subtle subclinical changes in coagulation tests to clinically overt bleeding or thrombosis. It is presumed that the thrombotic tendency due to coagulation factors and hypofibrinolysis is increased in hyperthyroidism, whereas hypothyroidism predilects to bleeding [1, 2]. Many studies showed that increased free thyroxine (fT4) was a risk factor for venous thrombosis, and contrastingly, low fT4 was protective against it [3]. However, in some published papers, it has been revealed that there is a hypercoagulable state due to the increase in some coagulation and fibrinolysis activation markers in hypothyroidism $[4,5]$. Moreover, even in patients with subclinical hypothyroidism, it has been shown that the risk of thrombosis is increased in contrast to the bleeding tendency $[6,7]$.

Thromboelastography (TEG) is a technique used to evaluate the tendency for bleeding or coagulation, which cannot be detected by routine laboratory tests. Rotational thromboelastometry (ROTEM), as a modified version of TEG, has emerged as a technique for in vitro evaluation of clot firmness, clot formation, and fibrinolysis in complete blood samples. The viscoelastic and mechanical features of clots are measured using ROTEM by collecting information about various steps of coagulation. Thus, the coagulation process can be evaluated globally and information regarding in vivo hemostasis can be 
obtained by laboratory means [8]. In the literature, there are studies using ROTEM/TEG to detect coagulation and bleeding disorders in trauma, postoperative bleeding, malignancy, ischemic heart disease, liver transplant, cardiac surgeries, and even inflammatory rheumatologic diseases[8-10]. The use of ROTEM/TEG to evaluate coagulation disorders in thyroid diseases is not common and has been used only in patients with thyroid cancer [11]. In the study, we aimed to evaluate the hemostatic system globally using ROTEM/TEG, which was never been used before to specifically investigate thrombotic tendency, and to establish the effects of possible disease-related factors on ROTEM/TEG parameters in thyroid diseases.

\section{Materials And Methods}

\section{Subjects}

Thirty patients with new-onset autoimmune-based hypothyroidism and 30 with hyperthyroidism before the commencement of therapy were enrolled in the study, along with 30 healthy volunteer subjects without thyroid disease. The normal range for thyroid function assays in our institution is thyroid-stimulating hormone (TSH) $0.35-4.94 \mu \mathrm{lU} / \mathrm{mL}$, free thyroxine (fT4) $0.81-1.42 \mathrm{ng} / \mathrm{mL}$, and free tri-iodothyronine (fT3) 1.71-3.71 pg/mL Hyperthyroidism was defined as fT $4>1.42 \mathrm{ng} / \mathrm{mL}$ and TSH $<0.35 \mu \mathrm{lU} / \mathrm{mL}$ and the overt hypothyroidism was defined as TSH $>4.94 \mathrm{mIU} / \mathrm{L}$ and $\mathrm{fT} 4<0.81 \mathrm{ng} / \mathrm{dL}$. Subjects with diabetes mellitus (DM); hypertension (HT); chronic renal failure (CRF); rheumatologic, hematologic, cardiopulmonary, or cerebrovascular disease; hematologic or solid organ malignancies; a history of venous or arterial thromboemboli; and current use of acetylsalicylic acid (ASA), non-steroid anti-inflammatory drugs (NSAIDs) or anti-coagulant/anti-aggregant drugs were excluded. In addition, women with non-thrombotic clinical signs such as thrombocytopenia, hemolytic anemia or livedo reticularis or with a history of maternal morbidity suggestive of clinical signs of antiphospholipid antibody syndrome were not included in the study. Age- and sex-matched healthy subjects enrolled; none had any chronic disease or current drug use. TEG analyses were performed in all patients with thyroid dysfunction before medical therapy and healthy volunteer subjects at the time of enrollment to the study. Informed consent was obtained from all individual participants included in the study.

TSH, fT4,fT3, thyroid antibodies [anti-thyroid peroxidase-anti-TPO (0-34 IU/mL), anti-thyroglobulin-anti-TG $(0-4,11 \mathrm{IU} / \mathrm{mL})$, thyrotropin receptor antibodies-TRAb (>1 positive)] were measured with an automated direct chemiluminescent immunoassay. Complete blood count (CBC) (Becton-Dickinson anticoagulated tubes), prothrombin time (PT) (performed using Noplastine Cl Plus (Diagnostica Stago, Asnieres, France), normal range: 9.4-15 seconds), activated partial thromboplastin time (PTT) (performed using rabbit brain cephalin with kaolin as the activator (Diagnostica Stago, Asnieres, France), normal range: 26-36 seconds), fibrinogen (STA®Fibrinogen kit was used for the quantitative determination of fibrinogen levels using the clotting method of Clauss (Diagnostica Stago, Asnieres, France), normal range: $200-400 \mathrm{mg} / \mathrm{dL}$ ), and ROTEM to detect the tendency to thrombosis, were simultaneously studied.

\section{ROTEM Study Method and Interpretation}


Blood samples were collected using a 19-gauge needle under minimum stasis. Blood samples for ROTEM and coagulation profile were drawn into 4.5 -mL vacutainers (Becton-Dickinson) containing $3.2 \%$ trisodium citrate with a citrate/blood ratio of 1:9. With the same procedure, more blood was drawn for a CBC into Becton-Dickinson anticoagulated tubes. The main principle of ROTEM study involves a motionless cuvette and a pin which is inserted into a certain amount of whole blood lying in the cuvette. The fibrin-thrombocyte bonds within this clot promote movement of the needle back and forth at a certain angle and finally convert the strength power to electrical signals with the help of an electrotransducer which are then transferred to a graphic document [8].

ROTEM analysis was performed using ROTEM device Coagulation Analyzer model Gamma 2500 (Tem International, Munich, Germany). The 300 microliter sodium citrated whole blood samples of the subjects were recalcified using $20 \mu \mathrm{l} 0.2 \mathrm{~mol} / \mathrm{L} \mathrm{CaCl}$ (starTEM ${ }^{\circledR} ;$ Pentapharm, Munich, Germany). Different activators $(20 \mu \mathrm{l}$ partial tromboplastin-phospholipid and ellagic acid containing rabbit brain extract as contact activator was used for intrinsic pathway activation, $20 \mu \mathrm{l}$ tissue factor containing rabbit brain extract was used for extrinsic pathway activation) were used for activation of intrinsic (I) and extrinsic (E) pathways were used. Therefore coagulation time (CT) (time from baseline amplitude until $2 \mathrm{~mm}$ rise seconds), clot formation time (CFT) (time interval from $2 \mathrm{~mm}$ rise until $20 \mathrm{~mm}$ rise - seconds) and maximum clot firmness (MCF - mm) were measured using tromboelastography. Shortened CT and/or CFT and/or prolonged MCF was considered as tendency for hypercoagulability $[9,10,12]$. These parameters were recorded seperately for both I and E pathways.

\section{Statistical Analysis}

The statistical analysis was done using SPSS version 20.0. Continuous variables were given as mean \pm standard deviation, while categorical variables as frequency and percentage. Distribution of normality was evaluated by Shapiro Wilk's test. For the comparison of normally distributed groups, the independent t-test was used if the group number was two, and one-way analysis of variance (ANOVA) was used if the group number was three. Tukey's multiple comparison tests were used for multiple comparisons of normally distributed groups. For the comparison of non-normally distributed groups, the Mann-Whitney $\mathrm{U}$ test was used for comparisons if the group number was two, and the Kruskal-Wallis $\mathrm{H}$ test was used if the group number was three. In correlation analysis, Pearson correlation analysis was used for normally distributed parameters and Spearman correlation coefficients for non-normally distributed parameters. A $p$ value $<0,05$ within $95 \%$ confidence interval was accepted as significant.

\section{Results}

The mean age of the 30 patients with hypothyroidism and 30 with hyperthyroidism was $46 \pm 15.9$ years and $44.8 \pm 13$ years, respectively. All patients' sera were positive for at least one of the thyroid autoantibodies (anti-TPO, anti-TG, TRAb). When the patients with thyroid dysfunction were compared with the healthy subjects, those with hypothyroidism had a higher number of thrombocytes and those with hyperthyroidism had both higher thrombocytes and fibrinogen levels $(p=0.004, p=0.001, p<0.001$, respectively). Laboratory and demographic datas results are given in Table 1. 
Table 1

Comparison of the demographic data and laboratory results of the hypothyroid, hyperthyroid, and healthy control groups

\begin{tabular}{|c|c|c|c|c|c|c|}
\hline & Hypothyroidism & Hyperthyroidism & Controls & $\mathrm{p}^{*}$ & $p+$ & p\# \\
\hline Age $\pm S D$, years & $46 \pm 15.9$ & $44.8 \pm 13$ & $41.5 \pm 13$ & 0.543 & 0.593 & 0.492 \\
\hline $\begin{array}{l}\text { Sex, } \\
\text { female/male }\end{array}$ & $17 / 13$ & $18 / 12$ & $20 / 10$ & - & - & - \\
\hline TSH, ulU/mL & $78.6 \pm 25.3$ & $0.0006 \pm 0.002$ & $1.69 \pm 1.03$ & - & - & $<0.01$ \\
\hline fT4, ng/dL & $0.56 \pm 0.28$ & $2.51 \pm 1.11$ & $1.26 \pm 0.16$ & - & - & $<0.01$ \\
\hline fT3, pg/mL & $1.32 \pm 0.32$ & $13.1 \pm 9.9$ & - & - & - & - \\
\hline PT, sec & $11.3 \pm 0.8$ & $11.9 \pm 1.0$ & $12.1 \pm 1.1$ & 0.044 & 0.724 & 0.53 \\
\hline PTT, sec & $31.4 \pm 3$ & $30.6 \pm 3$ & $29.7 \pm 2.6$ & 0.114 & 0.428 & 0.128 \\
\hline INR & $0.98 \pm 0.07$ & $1.02 \pm 0.08$ & $1.07 \pm 0.07$ & 0.001 & 0.06 & 0.001 \\
\hline $\begin{array}{l}\text { Fibrinogen } \pm S D \text {, } \\
\mathrm{mg} / \mathrm{dL}\end{array}$ & $338 \pm 66$ & $364 \pm 78$ & $301 \pm 56$ & 0.179 & 0.001 & 0.001 \\
\hline $\begin{array}{l}\text { Platelet } \\
\text { counts } \pm S D / \mu \mathrm{L}\end{array}$ & $287,826 \pm 88,967$ & $289,000 \pm 63,730$ & $224,533 \pm 44,389$ & 0.004 & $<0.001$ & $<0.001$ \\
\hline \multicolumn{7}{|c|}{ * Hypothyroid vs. healthy control } \\
\hline \multicolumn{7}{|c|}{ + Hyperthyroid vs. healthy control } \\
\hline \multicolumn{7}{|l|}{ \# Within groups } \\
\hline
\end{tabular}

According to the ROTEM/TEG results, the I-CT, E-CT, E-CFT, and I-CFT levels were not statistically significant in the hypo- and hyperthyroid groups in comparison with the healthy control group. Only the I-MCF and EMCF values were longer in both the hypo- and hyperthyroid groups compared with the healthy subjects. IMCF was $64.1 \pm 6.1 \mathrm{~mm}$ in the hypo- and $62.9 \pm 6.5 \mathrm{~mm}$ in the hyperthyroid group, whereas it was $58.9 \pm 4.9$ $\mathrm{mm}$ in the healthy group $(\mathrm{p}=0.014)$ (hypothyroid vs. healthy control groups), $\mathrm{p}=0.020$ (hyperthyroid vs. healthy control). In addition, E-MCF was $66.3 \pm 6 \mathrm{~mm}$ in the hypo- and $65.7 \pm 6.2 \mathrm{~mm}$ in the hyperthyroid group in comparison with $61.1 \pm 4.4 \mathrm{~mm}$ in the healthy control group $p=0.009$ (hypothyroid vs. healthy control), $p=0.003$ (hyperthyroid vs. healthy control). ROTEM/TEG results according to the groups are given in Table 2. 
Table 2

Comparison of thromboelastography results in the hypothyroid, hyperthyroid, and healthy control groups

\begin{tabular}{|c|c|c|c|c|c|c|}
\hline & Hypothyroidism & Hyperthyroidism & Controls & $\mathrm{p}^{*}$ & $\mathrm{p}+$ & p\# \\
\hline $\mathrm{I}-\mathrm{CT} \pm \mathrm{SD}$, sec & $210.3 \pm 34.2$ & $205 \pm 41.5$ & $195 \pm 38.8$ & 0.451 & 0.597 & 0.434 \\
\hline I-CFT $\pm S D$, sec & $87.4 \pm 26.4$ & $87.2 \pm 27.3$ & $101.2 \pm 32.6$ & 0.257 & 0.118 & 0.107 \\
\hline $\mathrm{I}-\mathrm{MCF} \pm S D, \mathrm{~mm}$ & $64.1 \pm 6.1$ & $62.9 \pm 6.5$ & $58.9 \pm 4.9$ & 0.014 & 0.020 & 0.006 \\
\hline $\mathrm{E}-\mathrm{CT} \pm \mathrm{SD}, \mathrm{sec}$ & $76.7 \pm 16.1$ & $90.8 \pm 26.6$ & $94.6 \pm 30.9$ & 0.075 & 0.833 & 0.083 \\
\hline $\mathrm{E}-\mathrm{CFT} \pm S \mathrm{~S}, \mathrm{sec}$ & $101.8 \pm 33.9$ & $105.7 \pm 34.2$ & $103 \pm 27$ & 0.993 & 0.931 & 0.892 \\
\hline $\mathrm{E}-\mathrm{MCF} \pm S \mathrm{D}, \mathrm{mm}$ & $66.3 \pm 6$ & $65.7 \pm 6.2$ & $61.1 \pm 4.4$ & 0.009 & 0.003 & 0.001 \\
\hline \multicolumn{7}{|c|}{ * Hypothyroid vs. healthy controls } \\
\hline \multicolumn{7}{|c|}{ + Hyperthyroid vs. healthy controls } \\
\hline \multicolumn{7}{|l|}{ \# Within groups } \\
\hline $\begin{array}{l}\text { I: intrinsic pathw } \\
\text { clot firmness, se }\end{array}$ & $\begin{array}{l}\text { extrinsic path } \\
\text { ond, SD; Stan }\end{array}$ & $\begin{array}{l}\text { TT; coagulation } \\
\text { leviation }\end{array}$ & CFT; clot & on & MCF & imum \\
\hline
\end{tabular}

\section{Correlation analyses}

In the correlation analyses, ROTEM/TEG parameters and age, TSH, free T4 and T3, and fibrinogen levels and thrombocyte count were evaluated in the hypothyroid, hyperthyroid, and healthy control groups, separately. The correlation analysis results that found to be statistically significant are given in Table 3 and shown in Figure 1 and 2.

Table 3

Correlation analysis results of ROTEM parameters

\begin{tabular}{|llllll|}
\hline & & I-CFT & I-MCF & E-CFT & E-MCF \\
\hline Hypothyroid group & Platelet counts & $r=-0.753$ & $r=0.584$ & $r=-0.538$ & $r=0.543$ \\
& & $p<0.0001$ & $p=0.001$ & $p=0.002$ & $p=0.002$ \\
\cline { 2 - 5 } & Fibrinogen levels & - & $r=0.401$ & - & $r=0.361$ \\
& & & $p=0.028$ & & $p=0.05$ \\
\hline Hyperthyroid Group & Platelet counts & $r=-0.482$ & $r=0.448$ & $r=-0.476$ & $r=0.529$ \\
& & $p=0.002$ & $p=0.004$ & $p=0.002$ & $p=0.001$ \\
\cline { 2 - 6 } & Fibrinogen levels & $r=-0.397$ & $r=0.412$ & $r=-0.476$ & $r=0.470$ \\
& & $p=0.012$ & $p=0.009$ & $p=0.002$ & $p=0.003$ \\
\hline I: intrinsic pathway; E; extrinsic pathway, CFT; clot formation time, MCF; maximum clot firmness.
\end{tabular}




\section{Discussion}

This study is the first in the literature to evaluate thrombotic tendency using the ROTEM/TEG laboratory method in thyroid diseases (hypo- and hyperthyroidism). We found significant results promoting thrombosis in ROTEM/TEG parameters, both in hypo- and hyperthyroidism.

Until recently, the majority of both experimental and clinical studies showed that hyperthyroidism was associated with an increased risk of thrombosis, and hypothyroidism with an increased bleeding tendency. However, the most recent studies showed a predilection to thrombosis in patients with hypothyroidism, similar to hyperthyroidism [7, 13-15]. Hemostasis is a complicated process involving thrombocytes, coagulation factors, and interaction with the endothelium. Thrombus formation and resolution depend on thrombocyte function, coagulability of blood, and vascular endothelium. Thrombosis formation in hyperthyroidism is associated with various coagulation factors, and a number of factors such as $\mathrm{HT}$, lipid disorders, and increased body weight may operate in thrombosis formation in hypothyroidism (both overt and subclinical hypothyroidism). Furthermore, it was shown that by also accelerating the atherosclerotic process, these stated factors might ultimately cause an increased risk of cardiovascular disorders, resulting in thromboemboli [15].

The effects of thyroid hormones and the relation with thrombocytes, coagulation factors, and fibrinogen levels have both been extensively assessed in studies regarding coagulation defects in thyroid diseases [3, $16,17]$. In these papers, decreased fibrinolytic activity and coagulation were detected in hyperthyroidism, and increased or decreased fibrinolytic activity were suggested in hyperthyroidism [18-20]. The classic hemostasis-coagulation tests used until recently including fibrinogen and factor levels do not reflect the whole, but certain components of the coagulation process [15]. ROTEM/TEG allows rapid in vitro dynamic and global evaluation of hemostasis. Additionally, it represents a rapid, portable, and verified method for physicians and may obviate thrombotic tendency undetectable in routine coagulation tests in various settings [8, 21]. In this study, we simply measured fibrinogen levels and thrombocyte counts probably responsible for the pathogenesis of thrombosis in thyroid diseases, and made a global assessment by incorporating many factors regarding hemostasis using ROTEM/TEG for the first time in the literature.

One of the most important components of the coagulation system is thrombocytes. Various thrombocyte changes were observed in overt hyperthyroidism. Homoncik et al. found increased thrombocyte function in patients with hyperthyroidism compared with euthyroid control subjects [22, 23]. In our study, the thrombocyte count increased in patients with hypo- and hyperthyroid groups in comparison with the healthy control group. MCF, as a ROTEM parameter, which is affected by fibrinogen concentration, levels of factor $\mathrm{XIII}$, thrombocyte count, thrombocyte function, and thrombin concentration. A significant correlation between MCF and thrombocyte count has been shown in many diseases [10, 12, 24]. It can be claimed that thrombocytes constitute an important component of thrombotic tendency shown by ROTEM/TEG in patient groups with thyroid dysfunction because the thrombocyte count was significantly correlated with both intrinsic and extrinsic ROTEM/TEG tests in both thyroid disease groups of our study. 
Another important component of the coagulation system is fibrinogen, which contributes to thrombosis risk in various ways. Although the underlying mechanisms are obscure, it is assumed that the situation is probably due to inflammation and hypercoagulability. High fibrinogen levels promote a hypercoagulable state by increasing thrombocyte aggregation and thrombocyte reactivity. Additionally, fibrin structure is affected by fibrinogen levels. In vitro fibrin clots in the presence of high fibrinogen concentrations are more dense, composed of thicker fibrin fibers, and are more resistant to fibrinolysis than clots formed in low fibrinogen concentrations [25]. Decreased fibrinolytic activity was also detected in hyperthyroidism due to increased levels of factor VIII, IX, von Willebrand factor (VWF), fibrinogen, and plasminogen activator inhibitor-1 (PAl-1) [15]. This finding was observed both in overt and subclinical hyperthyroidism due to endogenous and exogenous causes. The issue is more complicated in hypothyroidism. Hypercoagulation was observed in some studies involving patients with overt hypothyroidism, whereas hypercoagulation was present in the others. In the studies where hypercoagulation was evident, several causes including hypofibrinolysis, increased fibrinogen and PAl-1 levels, and decreased factor VIII and X activity were blamed as possible causes of hypercoagulability in hypothyroidism $[13,16,17]$. In accordance with other studies, we observed higher fibrinogen levels in the hyperthyroid group compared with the hypothyroid and healthy control groups. Although not statistically significant, the hypothyroid group had higher fibrinogen levels, similar to the hyperthyroid group, when compared with the healthy control group. We detected a thrombotic tendency by interrelation between fibrinogen and ROTEM parameters in both the hypo- and hyperthyroid groups. Despite conflicting studies regarding the tendency for hypercoagulability in hypothyroidism, we showed thrombotic tendency using ROTEM/TEG for the first time in hypothyroidism in agreement with the studies by Akıncı et al. and Cantürk et al. The positive correlation between fibrinogen levels and MCF as a ROTEM/TEG parameter in both groups supported thrombotic tendency and demonstrated that fibrinogen similar to thrombocytes was another important component effective on MCF $[7,13]$.

In addition to the changes in coagulation factors, abnormalities in thyroid hormones were also suggested as possible causes of coagulation abnormalities in thyroid diseases $[15,17]$. The relation between thyroid hormones and normal hemostasis is complicated, and how thyroid dysfunction affects hemostasis has not been definitively elucidated. Furthermore, it is claimed that underlying inflammation or autoimmunity may have effects on thrombosis $[2,26]$. Thyroid hormones may affect many physiologic and pathologic processes including blood coagulation and atherogenesis [15]. The role of the immune system in the hemostatic system was emphasized, and it was suggested that an activated immune system had the main effect on coagulation system because a similar influence was detected on hemostatic parameters in both subclinical and overt thyrotoxicosis [2]. Another study in 2010 supported the claim by showing the simultaneous presence of autoimmune-based antiphospholipid syndrome leading to thrombosis and thyroid autoimmunity [26]. In our study, no correlation was detected between thyroid hormones and ROTEM/TEG parameters. Furthermore, the thrombotic tendency in ROTEM/TEG parameters in both deficiency (hypothyroidism) and excess (hyperthyroidism) of thyroid hormones indicated that the level of thyroid hormones was not associated with this setting. Underlying autoimmunity or inflammation (inflammation-related thrombocyte increase) must be kept in mind as the possible main factor in the pathophysiology of thrombotic tendency evident by laboratory tests because autoimmune-based thyroid diseases were present in both groups. 
TEG studies in thyroid diseases are scarce in the literature, and to the best of our knowledge, only one study enrolled 62 patients with nodular goiter, 53 with papillary thyroid cancer, and 61 healthy subjects. In this study, TEG parameters [K (clot formation time), MA (final clot firmness), and angle (alfa) and R] were compatible with hypercoagulability in patients with papillary cancer before undergoing surgery. However, information regarding the hormonal status of the patients, i.e. eu-/hyper-/hypothyroidism, was missing. Thrombocyte count was higher in the papillary cancer group compared with the healthy group (statistically non-significant); thrombocyte count was correlated with some of TEG parameters [11]. Similarly, a positive correlation was present between thrombocyte count and ROTEM/TEG parameters in our study indicating thrombotic tendency. Nevertheless, our study differs from that study by being the first TEG/ROTEM study to include patients with hypo- and hyperthyroidism due to thyroid diseases other than thyroid malignancies.

Our study has some limitations. The first is the cross-sectional design. Serial analyses and long-term follow-ups will shed light on the possible pathophysiologic causes of coagulation defects in thyroid diseases. Secondly, because we did not follow up with the patients, the course of those with impaired ROTEM results remained unknown. Thirdly, the lipid profile as a possible risk factor for thrombosis was not studied in patients with hypothyroidism. Fourth, anti-phospholipid antibodies, which may be associated with thrombosis in autoimmune thyroid diseases, should not be considered as a laboratory test in asymptomatic patients. In addition, the results regarding the presence of asymptomatic antiphospholipid antibodies in autoimmune thyroid diseases are contradictory. Although this relationship is mentioned especially in Graves' patients, there are also publications showing that the frequency of asymptomatic antiphospholipid antibodies in autoimmune thyroid patients is not different from healthy individuals [27]. Lastly, we failed to include patients with thyroid dysfunction due to non-autoimmune causes as a control group. Despite all these drawbacks, thrombotic tendency according to ROTEM results in both hypo- and hyperthyroidism will contribute to the current literature.

As a result, our study is the first in the literature in which TEG/ROTEM was used in thyroid dysfunction and we found thrombotic tendency independent from thyroid hormones in patients with both hypo- and hyperthyroidism in the absence of clinical evidence of thrombosis. The correlation between ROTEM parameters and indirect measures of inflammation, and thrombocyte counts and fibrinogen levels as the main constituents of thrombosis during hemostasis may indicate that the major factor is inflammation (autoimmunity), rather thyroid hormones. Assessing the hemostasis profile of patients with both hypo- and hyperthyroidism may help to predict the future risk of clinical thromboembolism and thus help with patient management. Use of tests such as ROTEM/TEG in thyroid diseases may help physicians for globally assessing tendency for thrombosis and bleeding.

\section{Financial disclosure}

None

\section{Declarations}

Financial disclosure: None 
Ethical approval: Ethics committee was approved the study (Eskişehir Osmangazi University Ethical Board of Clinical Researches in 9th May 2019/no 07).

Conflicts of Interest: There is no confict of interests about the publication of this paper.

Informed consent: The need for written informed consent was waived considering the retrospective nature of the study.

Consent for publication: This study only collected the patient's gender, age, and laboratory data and did not involve the patient's personal privacy.

\section{Author Contributions}

GBC: conception and design, organization of the study, data processing, manuscript drafting, and writing the final version.

DUC: data collection and analyses, manuscript drafting, critical revision of the work and final version of the manuscript.

BT: data collection and analyses, manuscript drafting and revision.

HUT: data collection and analyses, manuscript drafting and revision.

\section{References}

1. Franchini M, Vescovi PP, Manzato F, Favaloro EJ. Hormones, endocrine disorders, and hemostasis. Semin Thromb Hemost. 2011;37(1):3-5.

2. Stuijver DJ, van Zaane B, Romualdi E, Brandjes DP, Gerdes VE, Squizzato A. The effect of hyperthyroidism on procoagulant, anticoagulant and fibrinolytic factors: a systematic review and metaanalysis. Thromb Haemost. 2012;108(6):1077-88.

3. van Zaane B, Squizzato A, Huijgen R, et al. Increasing levels of free thyroxine as a risk factor for a first venous thrombosis: a case-control study. Blood. 2010;115(22):4344-9.

4. Erem $\mathrm{C}$, Kavgaci $\mathrm{H}$, Ersoz $\mathrm{HO}$, et al. Blood coagulation and fibrinolytic activity in hypothyroidism. Int $\mathrm{J}$ Clin Pract. 2003;57(2):78-81.

5. Muller B, Tsakiris DA, Roth CB, Guglielmetti M, Staub JJ, Marbet GA. Haemostatic profile in hypothyroidism as potential risk factor for vascular or thrombotic disease. Eur $\mathrm{J}$ Clin Invest. 2001;31(2):131-7.

6. Erem C. Blood coagulation, fibrinolytic activity and lipid profile in subclinical thyroid disease: subclinical hyperthyroidism increases plasma factor X activity. Clin Endocrinol (Oxf). 2006;64(3):3239. 
7. Canturk Z, Cetinarslan B, Tarkun I, Canturk NZ, Ozden M, Duman C. Hemostatic system as a risk factor for cardiovascular disease in women with subclinical hypothyroidism. Thyroid. 2003;13(10):971-7.

8. Whiting D, DiNardo JA. TEG and ROTEM: technology and clinical applications. Am J Hematol. 2014;89(2):228-32.

9. Akay OM, Ustuner Z, Canturk Z, Mutlu FS, Gulbas Z. Laboratory investigation of hypercoagulability in cancer patients using rotation thrombelastography. Med Oncol. 2009;26(3):358-64.

10. Turk SM, Cansu DU, Teke HU, et al. Can we predict thrombotic tendency in rheumatoid arthritis? A thromboelastographic analysis (with ROTEM). Clin Rheumatol. 2018;37(9):2341-9.

11. Lu S, Kang R, Wang Y, et al. Altered TEG Parameters Identify Hypercoagulablilty and are of Diagnosis Value for Papillary Thyroid Carcinoma Patients. Exp Clin Endocrinol Diabetes. 2020;128(5):297-302.

12. Goren Sahin D, Sahin A, Akay OM. Comparison of Rotational Thromboelastography Findings in Pseudoexfoliation Syndrome Patients and Healthy Controls. J Glaucoma. 2016;25(11):879-82.

13. Akinci B, Comlekci A, Ozcan MA. The alteration of coagulation in patients with thyroid dysfunction. Recent Pat Endocr Metab Immune Drug Discov. 2011;5(1):50-7.

14. Kootte RS, Stuijver DJ, Dekkers OM, et al. The incidence of venous thromboembolism in patients with overt hyperthyroidism: a retrospective multicentre cohort study. Thromb Haemost. 2012;107(3):41722.

15. van Zaane B, Stuijver DJ, Squizzato A, Gerdes VE. Arterial and venous thrombosis in endocrine diseases. Semin Thromb Hemost. 2013;39(5):489-95.

16. Coban E, Aydemir M. Levels of plasma fibrinogen and D-dimer in subjects with subclinical hyperthyroidism. Med Sci Monit. 2008;14(1):CR42-6.

17. Dorr M, Robinson DM, Wallaschofski H, et al. Low serum thyrotropin is associated with high plasma fibrinogen. J Clin Endocrinol Metab. 2006;91(2):530-4.

18. Li Y, Chen H, Tan J, Wang X, Liang H, Sun X. Impaired release of tissue plasminogen activator from the endothelium in Graves' disease - indicator of endothelial dysfunction and reduced fibrinolytic capacity. Eur J Clin Invest. 1998;28(12):1050-4.

19. Chadarevian R, Bruckert E, Leenhardt L, Giral P, Ankri A, Turpin G. Components of the fibrinolytic system are differently altered in moderate and severe hypothyroidism. J Clin Endocrinol Metab. 2001;86(2):732-7.

20. Rennie JA, Bewsher PD, Murchison LE, Ogston D. Coagulation and fibrinolysis in thyroid disease. Acta Haematol. 1978;59(3):171-7.

21. Stanford SN, Sabra A, Lawrence M, et al. Prospective evaluation of blood coagulability and effect of treatment in patients with stroke using rotational thromboelastometry. J Stroke Cerebrovasc Dis. 2015;24(2):304-11.

22. Homoncik M, Gessl A, Ferlitsch A, Jilma B, Vierhapper H. Altered platelet plug formation in hyperthyroidism and hypothyroidism. J Clin Endocrinol Metab. 2007;92(8):3006-12.

23. Franchini M, Lippi G, Targher G. Hyperthyroidism and venous thrombosis: a casual or causal association? A systematic literature review. Clin Appl Thromb Hemost. 2011;17(4):387-92. 
24. Jeong SM, Song JG, Seo H, Choi JH, Jang DM, Hwang GS. Quantification of Both Platelet Count and Fibrinogen Concentration Using Maximal Clot Firmness of Thromboelastometry During Liver Transplantation. Transplant Proc. 2015;47(6):1890-5.

25. de Moerloose P, Boehlen F, Neerman-Arbez M. Fibrinogen and the risk of thrombosis. Semin Thromb Hemost. 2010;36(1):7-17.

26. Franchini M, Lippi G, Manzato F, Vescovi PP. Thyroid-associated autoimmune coagulation disorders. J Thromb Thrombolysis. 2010;29(1):87-91.

27. Mecacci F, Parretti E, Cioni R, et al. Thyroid autoimmunity and its association with non-organ-specific antibodies and subclinical alterations of thyroid function in women with a history of pregnancy loss or preeclampsia. J Reprod Immunol. 2000;46(1):39-50.

\section{Figures}
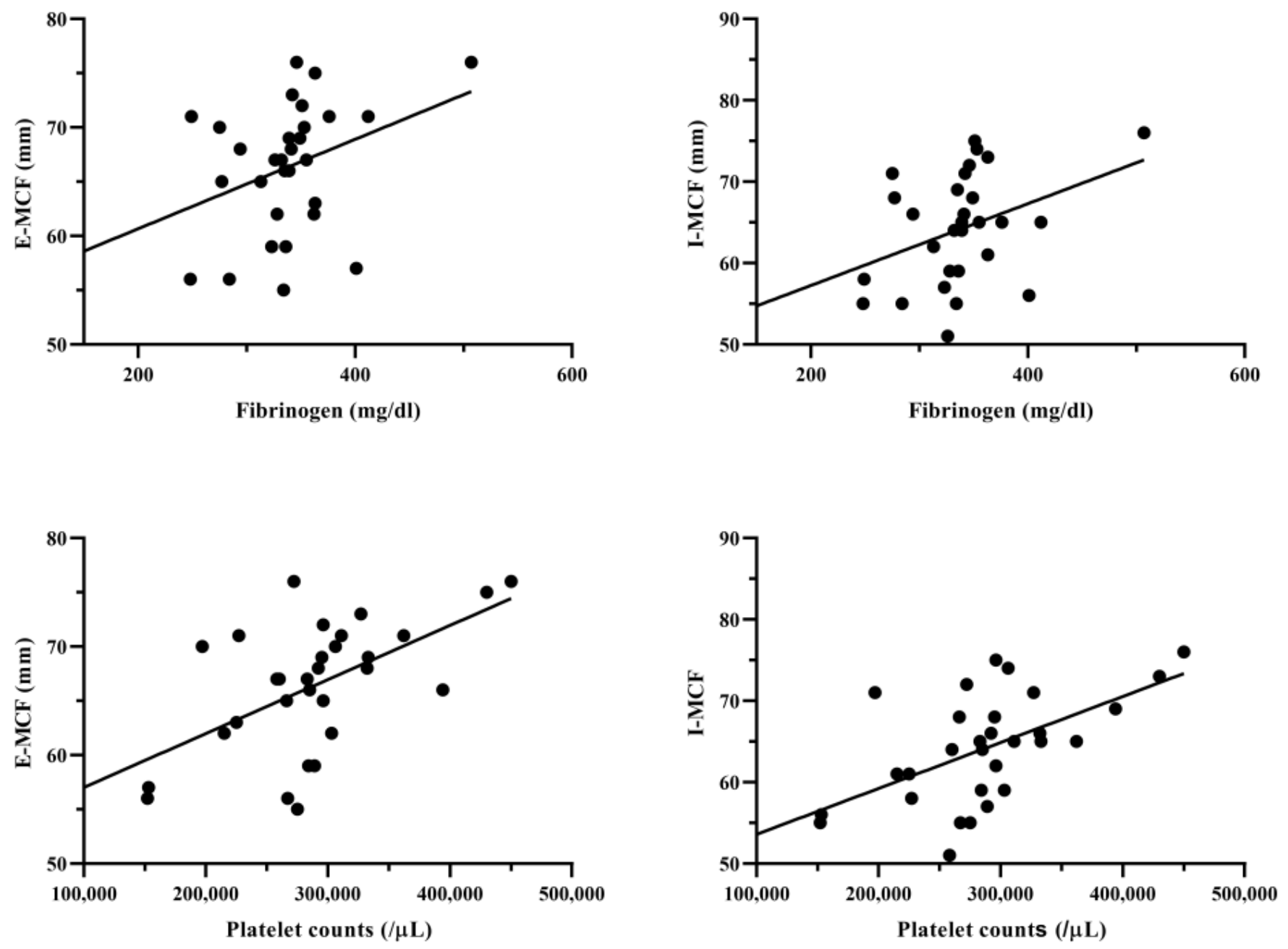

Figure 1 
Correlation analyses between intrinsic and extrinsic MCF levels and fibrinogen level and thrombocyte counts in patients with hypothyroidism.
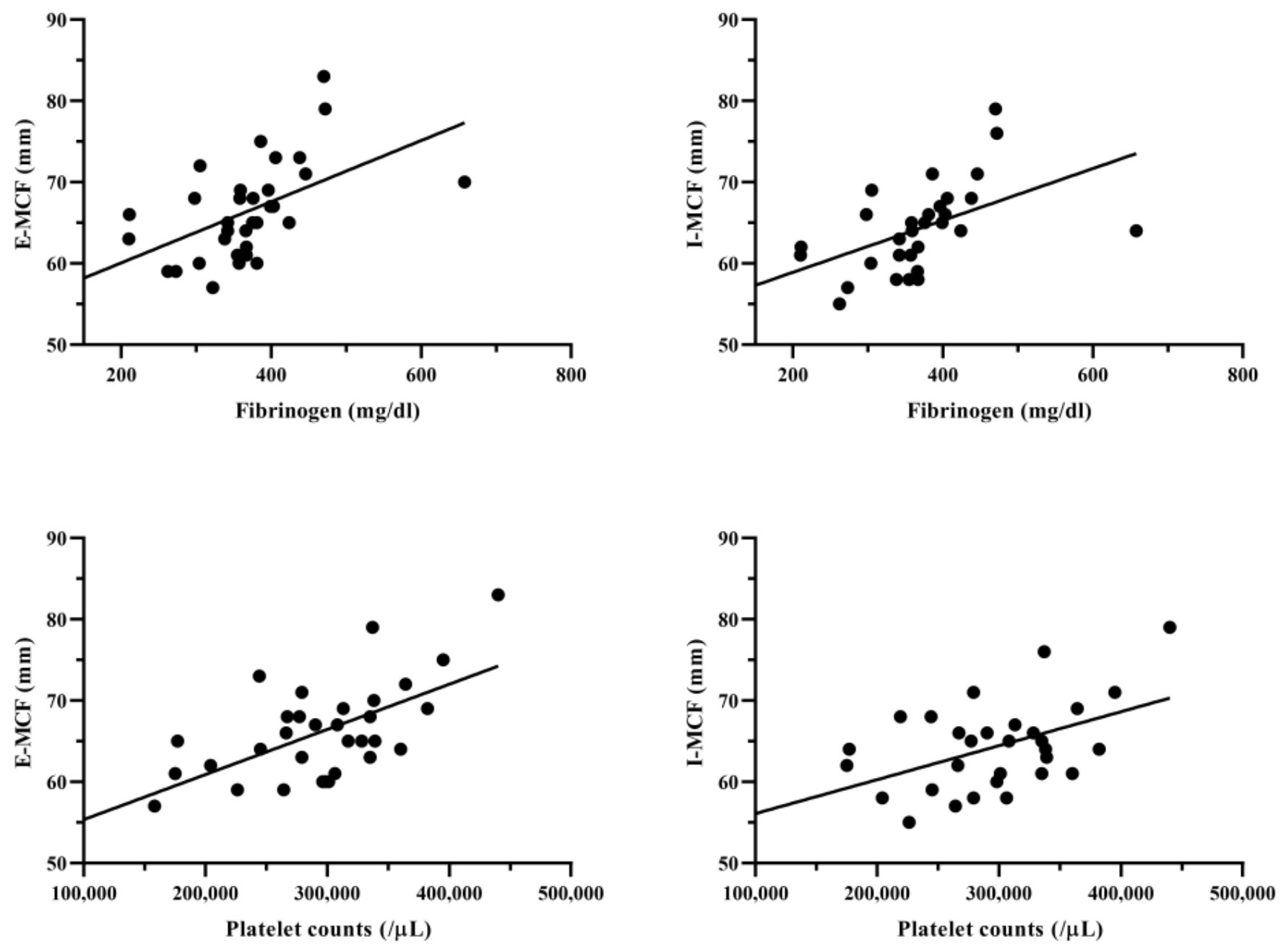

Figure 2

Correlation analyses between intrinsic and extrinsic MCF levels and fibrinogen level and thrombocyte count in patients with hyperthyroidism. 